\title{
Pushing the limits of whole genome amplification: successful sequencing of RADseq library from a single microhymenopteran (Chalcidoidea, Trichogramma)
}

Astrid Cruaud ${ }^{\text {Corresp.., }}{ }^{1}$ ， Géraldine Groussier ${ }^{2}$, Guenaëlle Genson ${ }^{1}$, Laure Sauné ${ }^{1}$, Andrew Polaszek ${ }^{3}$, JeanYves Rasplus ${ }^{1}$

${ }^{1}$ CBGP, INRA, CIRAD, IRD, Montpellier SupAgro, Univ Montpellier, Montpellier, France

2 Institut Sophia Agrobiotech, INRA, CNRS, Université Côte d'Azur, Sophia Antipolis, France

3 Department of Entomology, Natural History Museum, London, United Kingdom

Corresponding Author: Astrid Cruaud

Email address: astrid.cruaud@inra.fr

A major obstacle to high-throughput genotyping of microhymenoptera is their small size. As species are difficult to discriminate, and because complexes may exist, the sequencing of a pool of specimens is hazardous. Thus, one should be able to sequence pangenomic markers (e.g. RADtags) from a single specimen. To date, whole genome amplification (WGA) prior to library construction is still a necessity as at most 10ng of DNA can be obtained from single specimens (sometimes less). However, this amount of DNA is not compatible with manufacturer's requirements for commercial kits. Here we test the accuracy of the GenomiPhi kit V2 on Trichogramma wasps by comparing RAD libraries obtained from the WGA of single specimens (F0 and F1 generation, about1 ng input DNA for the WGA (0.17 - $2.9 \mathrm{ng})$ ) and a biological amplification of genomic material (the pool of the progeny of the F1 generation). Globally, we found that $99 \%$ of the examined loci (up to 48,189 for one of the crosses, $109 \mathrm{bp}$ each) were compatible with the mode of reproduction of the studied model (haplodiploidy) and Mendelian inheritance of alleles. The remaining $1 \%(0.01 \%$ of the analysed nucleotides) could represent WGA bias or other experimental / analytical bias. This study shows that the multiple displacement amplification method on which the GenomiPhi kit relies, could also be of great help for the high-throughput genotyping of microhymenoptera used for biological control, or other organisms from which only a very small amount of DNA can be extracted, such as human disease vectors (e.g. sandflies, fleas, ticks etc.). 
1 Pushing the limits of whole genome amplification: successful sequencing of RADseq library

2 from a single microhymenopteran (Chalcidoidea, Trichogramma)

3 Astrid Cruaud $^{1 *}$, Géraldine Groussier ${ }^{2}$, Guenaëlle Genson $^{1}$, Laure Sauné ${ }^{1}$, Andrew Polaszek ${ }^{3}$, 4 and Jean-Yves Rasplus ${ }^{1}$

5

$6{ }^{1}$ CBGP, INRA, CIRAD, IRD, Montpellier SupAgro, Univ Montpellier, Montpellier, France

$7 \quad{ }^{2}$ Institut Sophia Agrobiotech, INRA, CNRS, Université Côte d'Azur - 400, route des chappes -

8 BP 167 - 06903 Sophia Antipolis Cedex - France

$9 \quad{ }^{3}$ Department of Entomology, Natural History Museum, London SW7 5BD, UK

$12 *$ corresponding author : astrid.cruaud@inra.fr

\section{Abstract}

15 A major obstacle to high-throughput genotyping of microhymenoptera is their small size. As

16 species are difficult to discriminate, and because complexes may exist, the sequencing of a pool

17 of specimens is hazardous. Thus, one should be able to sequence pangenomic markers (e.g.

18 RADtags) from a single specimen. To date, whole genome amplification (WGA) prior to library

19 construction is still a necessity as at most 10ng of DNA can be obtained from single specimens

20 (sometimes less). However, this amount of DNA is not compatible with manufacturer's

21 requirements for commercial kits. Here we test the accuracy of the GenomiPhi kit V2 on

22 Trichogramma wasps by comparing RAD libraries obtained from the WGA of single specimens

23 (F0 and F1 generation, about $1 \mathrm{ng}$ input DNA for the WGA $(0.17-2.9 \mathrm{ng})$ ) and a biological 
24 amplification of genomic material (the pool of the progeny of the F1 generation). Globally, we 25 found that $99 \%$ of the examined loci (up to 48,189 for one of the crosses, 109 bp each) were 26 compatible with the mode of reproduction of the studied model (haplodiploidy) and Mendelian 27 inheritance of alleles. The remaining $1 \%(0.01 \%$ of the analysed nucleotides $)$ could represent 28 WGA bias or other experimental / analytical bias. This study shows that the multiple 29 displacement amplification method on which the GenomiPhi kit relies, could also be of great 30 help for the high-throughput genotyping of microhymenoptera used for biological control, or

31 other organisms from which only a very small amount of DNA can be extracted, such as human

32 disease vectors (e.g. sandflies, fleas, ticks etc.).

34 Running Head: RADseq from small DNA amount through WGA 


\section{INTRODUCTION}

37 Parasitoid wasps (especially Chalcidoidea; Heraty et al. 2013) are increasingly used as biocontrol agents of many crop pests to reduce pesticide use (Austin et al. 2000). Among them, minute wasps of the genus Trichogramma (210 species worldwide, 40 in Europe), which develop within the eggs of 200 species of moths damaging crops (e.g.

41 apple, banana, grape, maize, pine, tomato ; Consoli et al. 2010; Polaszek et al. 2012) are the most commercialized worldwide.

43 It is acknowledged that successful and safe biological control depends on accurate genetic and 44 phenotypic characterization of the strains released. Furthermore, host preferences and the potential of strains to hybridize with each other, or with native species, should be carefully studied. This is critical to avoid non-target effects such as gene introgression with indigenous species (Van Driesche \& Hoddle 2016). However, probably because most species of chalcids are minute wasps (less than a few millimetres long, and as little as $0.16 \mathrm{~mm}$ ) and are difficult to identify to species by non-specialists, strains are often released without in-depth characterization. RADseq, the sequencing of hundreds of thousands of DNA fragments flanking restriction sites

51 (Miller et al. 2007) has been successfully used for population genetics or phylogeography

52 (Emerson et al. 2010) to infer relationships between closely (Jones et al. 2013; Nadeau et al.

53 2013; Wagner et al. 2013) or more distantly (Cruaud et al. 2014; Hipp et al. 2014) related species, 54 to detect hybridization processes (Eaton \& Ree 2013; Hohenlohe et al. 2011), to identify markers 55 under selection and detect genes that are candidates for phenotype evolution (Hohenlohe et al.

56 2010), or to better understand the genomic architecture of reproductive isolation (Gagnaire et al.

57 2013). Thus, sequencing RAD markers appears relevant for in-depth characterisation of

58 Trichogramma species and strains used in biocontrol. 
59 A major obstacle to RAD sequencing of oophagous parasitoids is their small size. Ideally, one

60 should be able to sequence RAD markers from a single specimen. Indeed, species complexes

61 exist that are difficult to identify based on morphology only (Al Khatib et al. 2014; Kenyon et al.

62 2015; Mottern \& Heraty 2014), which makes sequencing of a pool of specimens risky. However,

63 to date, the DNA amount obtained from single specimens is not sufficient enough to build a

64 RADseq library. Usually, for minute specimens, at most $10 \mathrm{ng}$ of DNA is obtained (often less),

65 whereas about 150ng of DNA is required to build a RADseq library from our experience, and

66 much more can be required by private companies. Performing whole genome amplification

67 (WGA) prior to library construction is thus a necessity. So far a few studies have formally

68 examined the accuracy of WGA methods, mostly on human DNA and either a few loci (Hosono

69 et al. 2003; Lovmar et al. 2003; Sun et al. 2005) or a higher number of SNPs and loci but always

70 with 10ng or more input DNA (Abulencia et al. 2006; Barker et al. 2004; Blair et al. 2015;

71 ElSharawy et al. 2012; Paez et al. 2004; Pinard et al. 2006). All studies have concluded that the

72 multiple displacement amplification method, MDA, (Dean et al. 2002; Lasken 2009), which

73 relies on isothermal DNA amplification using a high-fidelity polymerase bacteriophage phi29;

74 (Paez et al. 2004) and random hexamer primers to decrease amplification bias and increase

75 product size, is among the most accurate.

76 So far, only one study has quantified sequence bias that might result from WGA prior to double-

77 digest RAD sequencing, ddRADseq; (Peterson et al. 2012); a variant of RADseq that uses two

78 restriction enzymes to cut DNA instead of one enzyme and a DNA shearing system. In their

79 study, Blair et al. (2015) use the Qiagen REPLI-g Mini Kit and 100 ng of input DNA (as

80 requested by the kit) extracted from liver samples of specimens of the grey mouse lemur

81 (Microcebus murinus). They conclude that the kit does not introduce bias for i) SNP calling as 
82 compared to what is obtained from native DNA of the same samples or ii) genome coverage as

83 compared to the published genome of M. murinus. Here we test the accuracy of the

84 GE Healthcare Life Sciences ${ }^{\mathrm{TM}}$ illustra $^{\mathrm{TM}}$ GenomiPhi V2 for the WGA of single Trichogramma

85 wasps prior to RADseq library construction. As for the REPLI-g Mini Kit, WGA is performed

86 using the MDA. However, the GenomiPhi kit requires 10 times less DNA ( $1 \mu \mathrm{l}$ of input DNA at

$8710 \mathrm{ng} / \mu \mathrm{l}$ ) but still more than what can be extracted from single Trichogramma wasps. As a

88 consequence, we had to push the limits of the kit, increasing the risk of inconsistent or

89 unrepresentative amplification of the genome. To test the accuracy of the GenomiPhi kit in these

90 challenging conditions we took advantage of the mode of reproduction of Trichogramma wasps

91 (arrhenotokous parthenogenesis; i.e. females develop from fertilized eggs and are diploid, while

92 males develop from unfertilized eggs and are haploid). We compared the number and sequences

93 of RAD tags obtained from the WGA of single individuals from the F0 and F1 generation with

94 the number and sequences of RAD tags obtained from the pool of their progeny (F2 generation)

95 (Fig 1). Thus, we compared RAD libraries obtained from a technical / artificial amplification

96 (WGA) and a biological / natural amplification (pool of specimens).

MATERIALS AND METHODS

101 Sampling and experimental design

102 The species Trichogramma brassicae Bezdenko, 1968 was used as model system. Specimens

103 were taken from the strain collection hosted by the Biological Resource Centre "Egg Parasitoid

104 Collection" (EP-Coll, Sophia-Antipolis, France) (Marchand et al. 2017) and confirmed as $T$. 
105 brassicae by AP and JYR using morphological characters detailed by Pino et al (2013),

106 especially the ratio between the length of the longest antennal seta and maximum antennal width.

107 Voucher specimens from this study are deposited permanently at the Natural History Museum,

108 London. Male (haploid) / female (diploid) pairs were placed in glass tubes and left free to mate

109 (1 pair per tube, Fig 1). Droplets of honey were provided as food, and eggs of Ephestia

110 kuehniella (Pyralidae) were used as hosts. F0 females and males were killed in 70\% ethanol

111 before emergence of the F1 generation. Emerging females of the F1 generation were kept

112 separated from males (no mating) and reared in new glass tubes (1 virgin female per tube).

113 Again, droplets of honey were provided as food and eggs of Ephestia kuehniella were used as

114 hosts. F1 females were killed before the emergence of the F2 generation, which was composed

115 only of males, as the reproductive strategy of T. brassicae is arrhenotokous parthenogenesis. Ten

116 parental crosses (F0 male x female) were attempted. For each cross, all F2 males were pooled

117 prior to DNA extraction. For each cross, a WGA was performed prior to RAD library

118 construction on the F0 female, the F0 male and one F1 female, while all F2 males were pooled

119 prior to DNA extraction and the resulting DNA was used directly as input for RAD library

120 construction without WGA. Thus, the F2 generation was used as a negative control. In

121 Trichogramma brassicae, females develop from fertilized eggs and are diploid, while males

122 develop from unfertilized eggs and are haploid. We took advantage of this reproductive strategy

123 to test for potential bias introduced by WGA. Indeed, with such a reproductive strategy, expected

124 results are as follows: for all RAD tags i) F0 males should be haploid, ii) F0/F1 females should

125 be diploid and either homozygous or heterozygous. We also expect that most RAD tags will

126 follow Mendelian laws of inheritance (Fig 1). We thus expect compatibility of

127 parental and offspring genotypes from the F0 to the F2 generation. Hence, for example, i) all 
128 SNPs found in the pool of F2 males should be present in the F0 generation; ii) F1 females should

129 be heterozygous when their parents possess different alleles.

130

131 DNA extraction and whole genome amplification

132 DNA extraction was performed with the Qiagen DNeasy 96 Blood \& Tissue Kit, following

133 manufacturer protocol with the following modifications to increase DNA yield: two successive

134 elutions (50 $\mu \mathrm{L}$ each) were performed with heated buffer $\mathrm{AE}\left(55^{\circ} \mathrm{C}\right)$ and an incubation step of 15

135 minutes followed by plate centrifugation (6000 rpm for 2 minutes).

136 DNA was quantified with a Qubit ${ }^{\circledR} 2.0$ Fluorometer (Invitrogen). To stay as close as possible to

137 the recommended amount / volume of DNA listed in the GenomiPhi protocol ( $1 \mu \mathrm{l}$ DNA input at

$13810 \mathrm{ng} / \mu \mathrm{l})$, ethanol precipitation of DNA was performed prior to WGA. 1/10 volume of sodium

139 acetate $3 \mathrm{M} \mathrm{pH} 5.2$ was added to the extract. Two volumes of cooled absolute ethanol were then

140 added to the mix which was then incubated at $-20^{\circ} \mathrm{C}$ overnight. The mix was then centrifuged

141 (30 min, $13000 \mathrm{rpm}, 4^{\circ} \mathrm{C}$ ) and the pellet was washed with $500 \mu \mathrm{l}$ of cooled $70 \%$ ethanol. After

142 another centrifugation $\left(15 \mathrm{~min}, 13000 \mathrm{rpm}, 4^{\circ} \mathrm{C}\right.$ ), the pellet was dried at room temperature and

143 resuspended in $4 \mu \mathrm{l}$ of sterile molecular biology ultrapure water, as a total resuspension of the

144 pellet would not have been obtained in a smaller volume. Concentrated DNA was quantified

145 with a Qubit ${ }^{\circledR}$ 2.0 Fluorometer (Invitrogen). DNA extracts were then subjected to WGA using

146 the GenomiPhi ${ }^{\mathrm{TM}}$ V2 DNA Amplification kit (GE Healthcare) with 1ul of concentrated DNA

147 used as input. The resulting DNA was quantified with a Qubit ${ }^{\circledR}$ 2.0 Fluorometer (Invitrogen).

148

\section{RADseq library construction}

150 Library construction followed Baird et al. (2008) and Etter et al. (2011) with modifications

151 detailed in Cruaud et al. (2014). The PstI enzyme was used as the cutter. The number of expected 
152 cut sites was estimated with an in silico digestion of the genome of $T$. pretiosum (assembly

153 Tpre_1.0, 196Mb) using a custom script. The quantity of P1 adapters $(100 \mathrm{nM})$ to be added to

154 saturate restriction sites (result $=3 \mathrm{uL}$ ) as well as the optimal time for DNA sonication on a

155 Covaris S220 ultrasonicator to obtain fragments of $300-600$ bp (results $=$ duty cycle $10 \%$,

156 intensity 5, cycles/burst 200, duration 70s) that are both specific to the studied group were

157 evaluated in a preliminary experiment. Briefly, RADseq libraries were built as follows: The

158 experiment to test the accuracy of WGA for RADseq of microhymenoptera is part of a larger

159 project that aims to resolve the phylogenetic relationships of European Trichogramma wasps.

160 Thus, more samples $(\mathrm{N}=40)$ than those used to answer our technical question were included in

161 the library. About 250ng of input DNA was used for each sample. After digestion with PstI,

162 samples were individually tagged with barcoded P1 adapters. Samples were then pooled eight by

163 eight. Five pools of eight samples were obtained. The DNA of each pool was sheared using a

164 Covaris ultrasonicator. After size selection on gel (300-600bp), end repair and 3'-end

165 adenylation, each pool was tagged with a different barcoded P2 adapter. A PCR enrichment step

166 was then performed. For each pool, five independent PCR reactions were used to increase

167 fragment diversity (10ng input DNA, 13 cycles, NEB Phusion High-Fidelity PCR Master Mix).

168 The five PCR products were pooled and purified with Ampure beads (Beckman Coulter). The

169 resulting enriched libraries $(\mathrm{N}=8)$ were quantified with Qubit, an Agilent Bioanalizer and qPCR

170 with the « Library Quantification Kit - Illumina/Universal » from KAPA (KK4824) and pooled

171 at equimolar ratio prior to sequencing. $2 * 125 \mathrm{nt}$ paired-end sequencing was performed at MGX-

172 Montpellier GenomiX on one lane of an Illumina HiSeq 2500 flow cell.

173

174 Data analysis 
175 Cleaning of raw data was performed with the wrapper RADIS (Cruaud et al. 2016) that relies on

176 Stacks (Catchen et al. 2013; Catchen et al. 2011) for demultiplexing of data and removing PCR

177 duplicates. Data analysis was performed with Stacks v1.46. Individual loci were built using

178 ustacks $[\mathrm{m}=3 ; \mathrm{M}=1 ; \mathrm{N}=2$; with the removal (r) and deleveraging (d) algorithms enabled]. Each

179 cross was analysed separately. Catalogs of loci were built with cstacks $(n=2)$. First, a catalogue

180 grouping F1 females and their progeny was built. Then a catalogue grouping samples from the

181 F0, F1 and F2 generation was built. sstacks was used to map individual loci to the catalogue.

182 rxstacks was then used to correct genotype and haplotype calls : i) Loci for which at least $50 \%$ of

183 the samples (when a pair composed of one F1 female and a pool of F2 males was analysed) or

$18425 \%$ of the samples (when F0, F1 and F2 were analysed together) had a confounded match to the

185 catalogue were removed; ii) excess haplotypes were pruned; iii) SNPs were recalled after

186 removal of possible sequencing errors using the bounded SNP model (--bound_high 0.1), and iv)

187 loci with an average log likelihood less than -10.0 were discarded. After this filtering step,

188 cstacks and sstacks were rerun. The program populations was then used to compare the RAD

189 tags obtained with or without WGA (parsing of the haplotypes.tsv and populations.log files).

190 Loci were kept only if i) they had a minimum stack depth of 10 and ii) all samples had a

191 sequence. Analyses were performed on the Genotoul Cluster (INRA, Toulouse).

192

193

194 RESULTS

195 On the ten attempted crosses, the number of F1 females varied from 0 to 38. Only three parental

196 crosses produced enough F2 males $(\mathrm{N}>100)$ to get a sufficient amount of DNA for RADseq

197 library construction without WGA. Consequently, RADseq libraries were constructed only on 
198 these crosses. DNA extraction of one third of the tested specimens provided an amount of DNA

199 that was below the detection limit of the Qubit (Table 1). WGA was not attempted on these

200 specimens. For other specimens, the average amount of DNA obtained with the Qiagen kit was

$20110.4 \mathrm{ng}(\min =6.2-\max =13.9)($ Table1 $)$. After DNA re-concentration, the average DNA

202 quantity used as input for the WGA was about $1.0 \mathrm{ng}(0.17-2.9 \mathrm{ng})$. On average, $947.5 \mathrm{ng}$ of

203 DNA was obtained with the WGA $(226-2393 \mathrm{ng})$.

204 In silico digestion of the genome of T. pretiosum revealed 59,433 PstI cut sites (i.e. 118,866

205 tags). An average of $2 * 3,757,867$ reads (109 bp) was obtained for the different samples after

206 quality filtering, demultiplexing and removal of PCR clones (Table 1). Two F1 females

207 (TRIC00027_1103 and TRIC00027_3103) were represented by much less reads than other

208 samples (595,204 and 1,991,305 respectively). The number of tags recovered by ustacks and

209 cstacks varied, but was comparable among the samples and in line with the predictions made on

210 the genome of $T$. pretiosum when these two females were excluded from calculation (average

211 number of ustacks tags $=132,787$; average number of cstacks tags $=128,293$, Table1).

212 The comparison of the loci obtained after filtering steps with rxstacks and populations revealed

213 that, on average, $97.6 \%$ of the loci were homozygous and identical in F1 females and the pool of

214 F2 males $(\min =96.8 \%-\max =98.2 \%$, Table 2$)$. On average, $0.7 \%(0.3 \%-1.3 \%)$ of the loci were

215 heterozygous and identical in F1 females and the pool of F2 males. Thus, there was a 98.3\%

$216(97.4 \%-99.0 \%)$ exact match between the loci of the F1 females (whose DNA was amplified with

217 WGA) and the whole progeny of the F1 generation (pool of males whose DNA was not

218 amplified with WGA). Between 1.0 and $2.6 \%$ of the loci were not identical between F1 females

219 and the pool of F2 males (Table 2). A careful inspection of the haplotypes revealed that about

$22060 \%$ of these differences could be explained by the experimental setup (Table 3 ). Indeed, RAD 
221 tags of a single female of the F1 generation are compared with RAD tags of the whole progeny

222 of the F1 generation (Fig 1). Globally, 99.3\% (98.9 - 99.6\%) of the shared loci were either

223 identical or displayed differences that could be explained by the experimental setup. The first

224 cross was used to check in detail the overall compatibility of the genotypes from the parental

225 generation (F0) with the whole progeny of the F1 generation (Table 4). 98.8\% of the 32,913 loci

226 shared by the four samples displayed haplotypes consistent with experimental setup and

227 Mendelian laws of inheritance (97.3\% of the loci were homozygous and identical between

228 samples). SNPs observed in 385 loci (which represent $1.2 \%$ of the loci and $0.01 \%$ of the

229 analysed nucleotides) were neither compatible with the mode of reproduction of the studied

230 model (haplodiploidy) nor Mendel's laws. Considering the haplotype observed in the pool of F2

231 males as a reference, questionable SNPs could be categorized into five categories as listed in

232 Table 5. In 90\% of the situations, SNPs found either in the F0 male (21\%), the F0 female

$233(32.5 \%)$ or the F1 female (36.6\%) were incompatible with haplodiploidy or with Mendelian

234 inheritance of alleles. 96 cases (about 25.0\%) represented situations where one allele was

235 missing for the F0 female or the F1 female to fit with Mendelian inheritance of alleles (possible

236 cases of allele drop-out).

238 DISCUSSION

239 Here we compare RAD libraries obtained from a technical / artificial amplification of DNA

240 (WGA of single specimen of micro-hymenoptera, F0 and F1 generations) and a biological /

241 natural amplification (pool of the progeny of the F1 generation). We push the limits of the kit

242 used for the WGA (GenomiPhi) by using 90\% less DNA (about 1.0ng) than the required amount

243 specified on the manufacturer's protocol (10ng). Globally, we show that $99 \%$ of the examined 
244 loci (up to 48,189; 109 bp each) were compatible with haplodiploidy and either identical among

245 specimens or compatible with Mendelian inheritance of alleles. These results are consistent with

246 observations by Blair et al. (2015) who used the Qiagen REPLI-g Mini Kit and 100 ng of input

247 DNA and showed that SNP calling between ddRAD libraries from native and amplified DNA

248 presented a $>98 \%$ match (up to 11,309 loci examined). They are also in agreement with older

249 studies that attempted to quantify bias induced by multiple displacement amplification method

250 (MDA) on which the GenomiPhi kit relies (> 99\% match; (Barker et al. 2004; ElSharawy et al.

251 2012; Paez et al. 2004), though with more input DNA (10ng).

252 With the exception of two samples, for which the construction of the library seems to have failed

253 (far fewer reads were obtained), comparable numbers of tags were obtained. This indicates that

254 the coverage of the genome is the same regardless whether native or amplified DNA is used as

255 suggested by previous studies on the potential bias induced by MDA (Abulencia et al. 2006;

256 Blair et al. 2015; Paez et al. 2004). Studies have suggested that WGA may induce allele dropout

257 especially when the starting amount of DNA is low $(<1 \mathrm{ng})$ (Handyside et al. 2004; Lovmar et al.

258 2003; Lovmar \& Syvänen 2006; Sun et al. 2005). El Sharawy et al. (2012) and Blair et al. (2015)

259 concluded that MDA had no significant effect on levels of homozygosity. Here about $1 \%$ of the

260 loci retained by our analytical pipeline (ie about $0.01 \%$ of the examined nucleotides) presented

261 problematic SNPs that were neither compatible with the biology of Trichogramma wasps nor

262 Mendelian inheritance of alleles. $0.3 \%$ of the SNPs were possible cases of allele drop-out (one

263 allele was missing for the F0 female or the F1 female to fit with Mendel's laws). A larger

264 sampling would be required to examine these few problematic SNPs in more detail. Here, a

265 correlation may exist between the number of problematic SNPs and the quantity of input DNA

266 used for the WGA [less bias in haploid F0 male (1.5 ng; 81 problematic SNPs; $0.002 \%$ of the 
267 examined nucleotides) as compared to F0 female ( $0.39 \mathrm{ng} ; 125 ; 0.003 \%)$ and F1 female ( $0.35 \mathrm{ng}$;

$268141 ; 0.004 \%)]$ but no definite conclusion can be drawn. It is noteworthy that if these

269 problematic SNPs can indeed result from bias caused by WGA, other explanations are possible

270 (competition between fragments for ligation of P1 adapters, mutation during enrichment PCR,

271 sequencing error). Indeed, although they are less frequent, bias are also observed in the pool of

272 F2 males (29 problematic SNPs; $0.0002 \%$ of the examined nucleotides). Finally, some

273 improvements could be made to our protocol. Extraction failed for a third of our specimens

274 (especially single males that are much smaller than females). Here we used the Qiagen kit 96-

275 well-plate format in order to facilitate the processing of many specimens simultaneously.

276 However, especially for valuable specimens, DNA yield could be increased with the spin-column

277 format, as higher centrifuge speed could be used. Furthermore, for projects that aim to target a

278 high number of specimens, re-concentration on SPRI beads may be used instead of using ethanol

279 precipitation of DNA. Indeed, such methods are compatible with robotic sample preparation.

280 However, while DNA yield could be better, working with very low amounts of buffer to

281 resuspend DNA could be troublesome.

282

283 CONCLUSION

284 In this study, we pushed the limits of the GenomiPhi kit V2 and successfully built RADseq

285 libraries from single micro-wasps (Trichogramma brassicae). Globally, we found that about

$28699 \%$ of the examined loci (up to 48,$189 ; 109$ bp each) were compatible with the mode of

287 reproduction of the studied model (haplodiploidy) and/or Mendelian inheritance of alleles. The

288 remaining $1 \%$ (about $0.01 \%$ of the analysed nucleotides) could represent WGA bias or other

289 experimental / analytical bias. It is noteworthy that the GenomiPhi kit V2 (and the new 
290 GenomiPhi kit V3) are affordable and easy to use by most laboratories, which is an important

291 point to consider given the increasing demand for the genomic characterisation of parasitoids

292 used in biocontrol programs or other disease-transmitting micro-arthropods (e.g. sand flies, fleas, 293 ticks etc.).

294

295 ACKNOWLEDGEMENTS

296 We are grateful to Montpellier GenomiX (Montpellier, France) for sequencing of the library and

297 to the Genotoul bioinformatics platform Toulouse Midi-Pyrenees for providing computing

298 resources. We thank two anonymous referees for their careful reading and valuable suggestions

299 to improve our manuscript.

301 REFERENCES

302 Abulencia CB, Wyborski DL, Garcia JA, Podar M, Chen W, Chang SH, Chang HW, Watson D,

303 Brodie EL, Hazen TC, and Keller M. 2006. Environmental whole-genome amplification

304 to access microbial populations in contaminated sediments. Applied and Environmental

$305 \quad$ Microbiology:3291-3301.

306 Al Khatib F, Fusu L, Cruaud A, Gibson G, Borowiec N, Ris N, Rasplus J-Y, and Delvare G. 2014. An integrative approach for species discrimination in the Eupelmus urozonus complex (Hymenoptera, Eupelmidae), with the descriptions of eleven new species from the West Palaearctic. Systematic Entomology 39:806-862.

310 Austin AD, Dowton M, and (Eds.). 2000. Hymenoptera: Evolution, Biodiversity and Biological Control: CSIRO PUBLISHING. 
312 Baird NA, Etter PD, Atwood TS, Currey MC, Shiver AL, Lewis ZA, Selker EU, Cresko WA,

313

314

315

316

317

318

319

320

321

322

323

324

325

326

327

328

329

330

331

332

333

and Johnson EA. 2008. Rapid SNP discovery and genetic mapping using sequenced RAD markers. PLoS ONE 3:e3376.

Barker DL, Hansen MS, Faruqi AF, Giannola D, Irsula OR, Lasken RS, Latterich M, Makarov V, Oliphant A, Pinter JH, Shen R, Sleptsova I, Ziehler W, and Lai E. 2004. Two methods of whole-genome amplification enable accurate genotyping across a 2320-SNP linkage panel. Genome Research 14:901-907.

Blair C, Campbell CR, and Yoder AD. 2015. Assessing the utility of whole genome amplified DNA for next-generation molecular ecology. Molecular Ecology Resources 15:10791090.

Catchen J, Hohenlohe PA, Bassham S, Amores A, and Cresko WA. 2013. Stacks: an analysis tool set for population genomics. Molecular Ecology 22:3124-3140.

Catchen JM, Amores A, Hohenlohe P, Cresko W, and Postlethwait JH. 2011. Stacks: Building and genotyping loci de novo from short-read sequences. G3-Genes Genomes Genetics $1: 171-182.10 .1534 / \mathrm{g} 3.111 .000240$

Consoli FL, Parra JRP, Zucchi RA, and (Eds.). 2010. Egg parasitoids in agroecosystems with emphasis on Trichogramma: Springer, Dordrecht.

Cruaud A, Gautier M, Galan M, Foucaud J, Sauné L, Genson G, Dubois E, Nidelet S, Deuve T, and Rasplus J-Y. 2014. Empirical assessment of RAD sequencing for interspecific phylogeny. Molecular Biology and Evolution 31:1272-1274.

Cruaud A, Gautier M, Rossi J-P, Rasplus J-Y, and Gouzy J. 2016. RADIS: Analysis of RAD-seq data for InterSpecific phylogeny Bioinformatics 32:3027-3028. 
334 Dean FB, Hosono S, Fang L, Wu X, Faruqi AF, Bray-Ward P, Sun Z, Zong Q, Du Y, Du J,

335 Driscoll M, Song W, Kingsmore SF, Egholm M, and Lasken RS. 2002. Comprehensive

336 human genome amplification using multiple displacement amplification. Proceedings of

337 the National Academy of Sciences of the United States of America 99:5261-5266.

338 Eaton DAR, and Ree RH. 2013. Inferring phylogeny and introgression using RADseq data: An example from flowering plants (Pedicularis: Orobanchaceae). Systematic Biology

El Sharawy A, Warner J, Olson J, Forster M, Schilhabel MB, Link DR, Rose-John S, Schreiber S, Rosenstiel P, Brayer J, and Franke A. 2012. Accurate variant detection across nonamplified and whole genome amplified DNA using targeted next generation sequencing. CM. 2010. Resolving postglacial phylogeography using high-throughput sequencing. Proceedings of the National Academy of Sciences of the United States of America 107:16196-16200. 10.1073/pnas.1006538107

Etter PD, Bassham S, Hohenlohe PA, Johnson EA, and Cresko WA. 2011. SNP discovery and genotyping for evolutionary genetics using RAD sequencing. In: Orgogozo V, and Rockman MV, eds. Molecular methods for evolutionary genetics: Humana Press, New York., 157-178.

Gagnaire PA, Normandeau E, Pavey SA, and Bernatchez L. 2013. Mapping phenotypic, expression and transmission ratio distortion QTL using RAD markers in the Lake Whitefish (Coregonus clupeaformis). Molecular Ecology 22:3036-3048. 
356 Handyside AH, Robinson MD, Simpson RJ, Omar MB, Shaw MA, Grudzinskas JG, and

357

358

359

360

361

362

363

364

365

366

367

368

369

370

371

372

373

374

375

376

377
Rutherford A. 2004. Isothermal whole genome amplification from single and small numbers of cells: a new era for preimplantation genetic diagnosis of inherited disease. Molecular Human Reproduction 10:767-772.

Heraty JM, Burks RA, Cruaud A, Gibson GAP, Liljeblad J, Munro J, Rasplus J-Y, Delvare G, Janšta P, Gumovsky A, Huber J, Woolley JB, Krogmann L, Heydon S, Polaszek A, Schmidt S, Darling DC, Gates MW, Mottern J, Murray E, Dal Molin A, Triapitsyn S, Baur H, Pinto JD, van Noort S, George J, and Yoder M. 2013. A phylogenetic analysis of the megadiverse Chalcidoidea (Hymenoptera). Cladistics 29:466-542.

Hipp AL, Eaton DAR, Cavender-Bares J, Fitzek E, Nipper R, and Manos PS. 2014. A framework phylogeny of the american oak clade based on sequenced RAD data. PLoS ONE 9:e93975. 10.1371/journal.pone.0093975

Hohenlohe PA, Amish SJ, Catchen JM, Allendorf FW, and Luikart G. 2011. Next-generation RAD sequencing identifies thousands of SNPs for assessing hybridization between rainbow and westslope cutthroat trout. Molecular Ecology Resources 11 (Suppl. 1):117122.

Hohenlohe PA, Bassham S, Etter PD, Stiffler N, Johnson EA, and Cresko WA. 2010. Population genomics of parallel adaptation in threespine stickleback using sequenced RAD tags. PloS Genetics 6:e1000862.

Hosono S, Faruqi AF, Dean FB, Du Y, Sun Z, Wu X, Du J, Kingsmore SF, Egholm M, and Lasken RS. 2003. Unbiased whole-genome amplification directly from clinical samples. Genome Research 13:954-964. 
378 Jones JC, Fan SH, Franchini P, Schartl M, and Meyer A. 2013. The evolutionary history of

379 Xiphophorus fish and their sexually selected sword: a genome-wide approach using 380 restriction site-associated DNA sequencing. Molecular Ecology 22:2986-3001.

$381 \quad 10.1111 / \mathrm{mec} .12269$

382 Kenyon SG, Buerki S, Hansson C, Alvarez N, and Benrey B. 2015. Uncovering cryptic 383 parasitoid diversity in Horismenus (Chalcidoidea, Eulophidae). PLoS ONE 10:e136063.

384 Lasken RS. 2009. Genomic DNA amplification by the multiple displacement amplification 385 (MDA) method. Biochemical Society Transactions 37:450.

386 Lovmar L, Fredriksson M, Liljedahl U, Sigurdsson S, and Syvänen AC. 2003. Quantitative evaluation by minisequencing and microarrays reveals accurate multiplexed SNP genotyping of whole genome amplified DNA. Nucleic Acids Research 31.

Lovmar L, and Syvänen AC. 2006. Multiple displacement amplification to create a long-lasting source of DNA for genetic studies. Human Mutation 27:603-614.

Marchand M, Sellier N, Warot S, Ion Scotta M, Ris N, and Groussier-Bout G. 2017. Formalisation d'un Centre de Ressources Biologiques dédié aux parasitoïdes oophages : CRB Ep-Coll. Cahier des Techniques de l'INRA Special Volume "Innovations

Mottern JL, and Heraty JM. 2014. Revision of the Cales noacki species complex (Hymenoptera, Chalcidoidea, Aphelinidae). Systematic Entomology 39:354-379. 
400 Nadeau NJ, Martin SH, Kozak KM, Salazar C, Dasmahapatra KK, Davey JW, Baxter SW, 401 Blaxter ML, Mallet J, and Jiggins CD. 2013. Genome-wide patterns of divergence and

402

403

404

405

406

407

408

409

410

411

412

413

414

415

416

417

418

419

420

421

422 gene flow across a butterfly radiation. Molecular Ecology 22:814-826. 10.1111/j.1365294X.2012.05730.x

Paez JG, Lin M, Beroukhim R, Lee JC, Zhao X, Richter DJ, Gabriel S, Herman P, Sasaki H, Altshuler D, Li C, Meyerson M, and Sellers WR. 2004. Genome coverage and sequence fidelity of $\varphi 29$ polymerase-based multiple strand displacement whole genome amplification. Nucleic Acids Research 32:e71. doi.org/10.1093/nar/gnh069

Peterson BK, Weber JN, Kay EH, Fisher HS, and Hoekstra HE. 2012. Double digest RADseq: an inexpensive method for de novo SNP discovery and genotyping in model and nonmodel species. PLoS ONE 7:e37135.

Pinard R, de Winter A, Sarkis GJ, Gerstein MB, Tartaro KR, Plant RN, Egholm M, Rothberg JM, and Leamon JH. 2006. Assessment of whole genome amplification-induced bias through high-throughput, massively parallel whole genome sequencing. BMC Genomics $7: 216$

Polaszek A, Rugman-Jones PF, Stouthamer R, Hernandez-Suarez E, Cabello T, and Pino Pérez M. del. 2012. Molecular and morphological diagnoses of five species of Trichogramma: biological control agents of Chrysodeixis chalcites (Lepidoptera: Noctuidae) and Tuta absoluta (Lepidoptera: Gelechiidae) in the Canary Islands. Biocontrol 57:21-35.

Pino, M del, Hernandez-Suarez, E, Cabello, T, Rugman-Jones, P, Stouthamer, R and Polaszek, A. 2013. Trichogramma canariensis (Insecta: Hymenoptera: Trichogrammatidae) a parasitoid of eggs of the twinspot moth Chrysodeixis chalcites (Lepidoptera: Noctuidae) in the Canary Islands. Arthropod Systematics and Phylogeny 71(3): 169-179. 
423 Sun G, Kaushal R, Pal P, Wolujewicz M, Smelser D, Cheng H, Lu M, Chakraborty R, Jin L, and

424 Deka R. 2005. Whole-genome amplification: relative efficiencies of the current methods. $425 \quad$ Legal Medicine 7:279-286.

426 Van Driesche R, and Hoddle MS. 2016. Non-target effects of insect biocontrol agents and trends 427 in host specificity since 1985. CAB Reviews 44.

428 Wagner CE, Keller I, Wittwer S, Selz OM, Mwaiko S, Greuter L, Sivasundar A, and Seehausen O. 2013. Genome-wide RAD sequence data provide unprecedented resolution of species boundaries and relationships in the Lake Victoria cichlid adaptive radiation. Molecular Ecology 22:787-798.

432

433

\section{DATA ACCESSIBILITY}

434 Cleaned reads are available as a NCBI Sequence Read Archive (\#SRP136713).

\section{TABLES AND FIGURES}

440 In Trichogramma, females develop from fertilized eggs and are diploid, while males develop 441 from unfertilized eggs and are haploid. In this figure, the coloured bars close to the female and 442 male symbols represent alleles. The breeding experiment was as follows: 1) A female/male pair

443 (F0 generation) was left free to mate in a glass tube (1 pair per tube). Sterilized eggs of Ephestia 444 kuehniella (Pyralidae) were provided for oviposition. 2) Only females of the F1 generation were 445 kept, males were discarded. Virgin F1 females were isolated into glass tubes (1 female per tube). 
446 Again, sterilized eggs of Ephestia kuehniella (Pyralidae) were provided for oviposition. 3) all F2

447 males were kept. This breeding experiment was replicated ten times. For clarity and to provide

448 allele frequency

449 predicted by Mendel's laws of inheritance, only two F1 females are represented on the figure.

450 Similarly, only two F2 males are represented for each F1 female. For each replicate, a WGA was

451 performed prior to RAD library construction on the F0 female, the F0 male and one F1 female,

452 while all F2 males were pooled prior to DNA extraction and the resulting DNA was directly used

453 as input for RAD library construction without WGA. Thus, the F2 generation was used as a

454 negative control. Photo Trichogramma brassicae (C) J.-Y. Rasplus

Table 1. Extraction, whole genome amplification and sequencing results

* reads obtained after demultiplexing and quality filtering with process_radtags; ** reads obtained after removal of PCR clones (input reads for the ustacks step); ${ }^{* * *}$ one catalog was built for each cross

460

Table 2. Pairwise comparison of loci obtained for females of the F1 generation and pools of males of the F2 generation. Analysed loci have been first corrected by rxstacks for genotype and haplotype calls and filtered with populations. Only loci that were present in the two samples 464 with a stack depth of 10 were kept.

Table 3. Summary of differences observed between F1 females and the whole progeny of 
470 Table 4. Comparison of loci obtained for the first crossing experiment. Analysed loci have

471 been first corrected by rxstacks for genotype and haplotype calls and filtered with populations.

472 Only loci that were present in the four samples with a stack depth of 10 were kept.

473

474 Table 5. Categories of SNPs not compatible with the mode of reproduction of the studied 475 model (haplodiploidy) or Mendel's laws of inheritance and number of occurrences of each

476 case. The different situations are illustrated by examples taken from the analysis of the 385

477 questionable SNPs.

478

479 


\section{Figure 1}

\section{Experimental setup}

In Trichogramma, females develop from fertilized eggs and are diploid, while males develop from unfertilized eggs and are haploid. In this figure, the coloured bars close to the female and male symbols represent alleles. The breeding experiment was as follows: 1) A female/male pair (F0 generation) was left free to mate in a glass tube (1 pair per tube). Sterilized eggs of Ephestia kuehniella (Pyralidae) were provided for oviposition.2) Only females of the F1 generation were kept, males were discarded. Virgin F1 females were isolated into glass tubes (1 female per tube). Again, sterilized eggs of Ephestia kuehniella (Pyralidae) were provided for oviposition. 3) all F2 males were kept. This breeding experiment was replicated ten times. For clarity and to provide allele frequency predicted by Mendel's laws of inheritance, only two F1 females are represented on the figure. Similarly, only two F2 males are represented for each F1 female. For each replicate, a WGA was performed prior to RAD library construction on the F0 female, the F0 male and one F1 female, while all F2 males were pooled prior to DNA extraction and the resulting DNA was directly used as input for RAD library construction without WGA. Thus, the F2 generation was used as a negative control. Photo Trichogramma brassicae[ J.-Y. Rasplus 

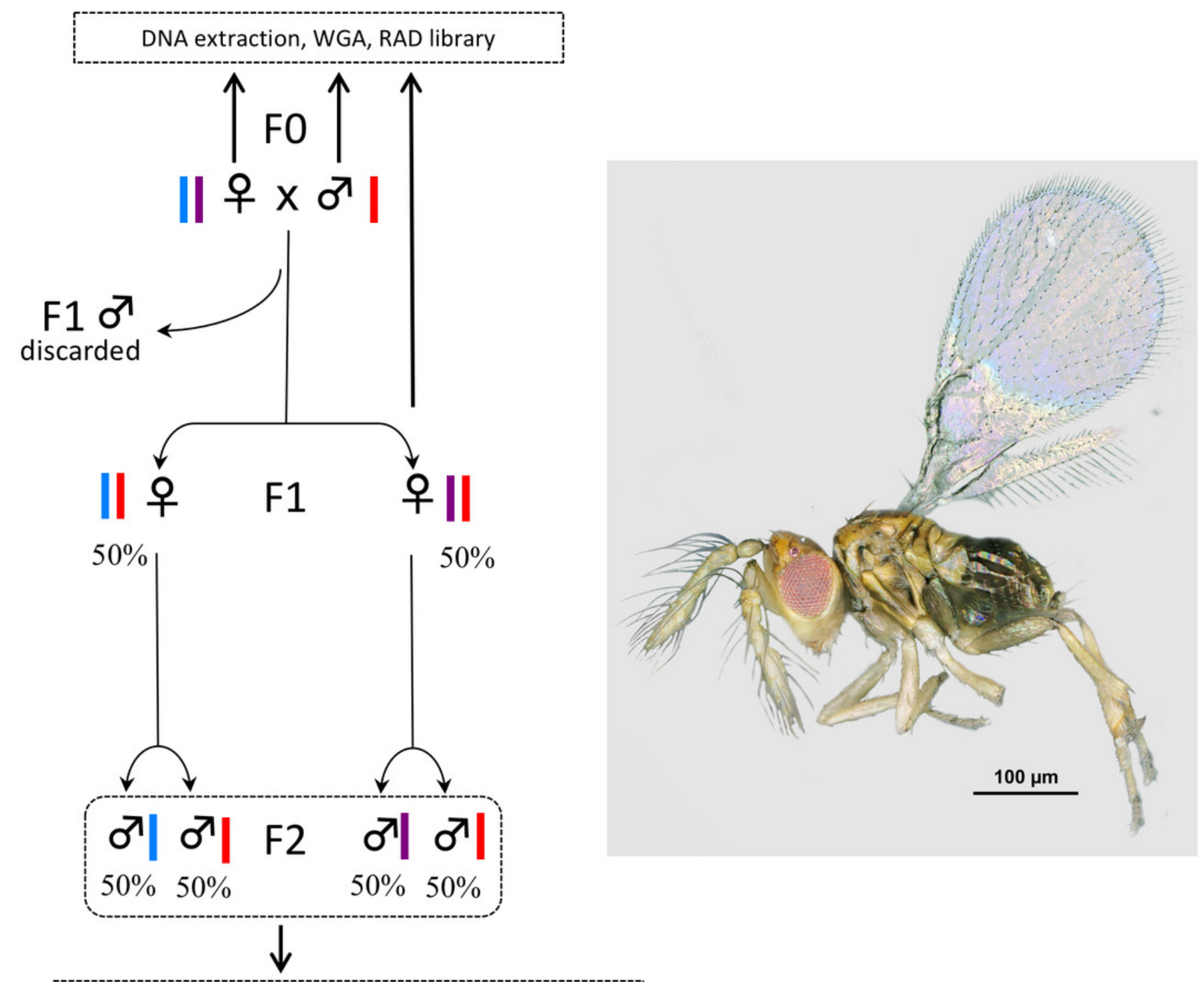

Pooling, DNA Extraction, no WGA, RAD library 


\section{Table $\mathbf{1}$ (on next page)}

Extraction, whole genome amplification and sequencing results

* reads obtained after demultiplexing and quality filtering with process_radtags; ** reads obtained after removal of PCR clones (input reads for the ustacksstep); *** one catalogue was built for each cross 
$1 \quad$ Table 1

\begin{tabular}{|c|c|c|c|c|c|c|c|c|c|c|}
\hline $\begin{array}{l}\text { Cross } \\
\#\end{array}$ & Sample code & Description & $\begin{array}{l}\text { qDNA } \\
\text { obtaine } \\
\text { d after } \\
\text { extracti } \\
\text { on (ng) }\end{array}$ & $\begin{array}{l}\text { qDNA } \\
\text { used for } \\
\text { WGA } \\
\text { (ng) }\end{array}$ & $\begin{array}{l}\text { qDNA } \\
\text { obtained } \\
\text { after WGA } \\
\text { (ng) }\end{array}$ & $\begin{array}{l}\text { Input } \\
\text { DNA } \\
\text { used for } \\
\text { RAD } \\
\text { library } \\
\text { (ng) }\end{array}$ & $\begin{array}{l}\text { Nb of } \\
\text { demultiplex } \\
\text { ed Reads* } \\
\text { (forward } \\
\text { only) }\end{array}$ & $\begin{array}{l}\text { Nb of } \\
\text { cleaned } \\
\text { reads** } \\
\text { (forward } \\
\text { only) }\end{array}$ & $\begin{array}{l}\text { ustacks : } \\
\text { Nb of loci }\end{array}$ & $\begin{array}{l}\text { ustacks: } \\
\text { Nb of } \\
\text { loci } * * *\end{array}$ \\
\hline 1 & TRIC00027_2101 & Male (F0), haploid, WGA & 11.5 & 1.5 & 500 & 169.0 & $6,774,680$ & $5,173,711$ & 136,623 & 130,060 \\
\hline 1 & TRIC00027_2102 & Female (F0), diploid, WGA & 10.6 & 0.39 & 1048 & 203.3 & $4,073,370$ & $3,179,891$ & 128,212 & 122,860 \\
\hline 1 & TRIC00027_2103 & Female (F1), diploid, WGA & 6.20 & 0.35 & 2393 & 281.2 & $4,597,505$ & $3,566,986$ & 130,565 & 124,845 \\
\hline 1 & TRIC00027_2199 & Pool of haploid males (F2) (n=933), no WGA & 735.4 & N.A. & N.A. & 269.0 & $4,818,385$ & $3,745,752$ & 127,709 & 125,047 \\
\hline 2 & TRIC00027_1101 & Male (F0), haploid, WGA & Too low & N.A. & N.A. & N.A. & N.A. & N.A. & N.A. & N.A. \\
\hline 2 & TRIC00027_1102 & Female (F0), diploid, WGA & Too low & N.A. & N.A. & N.A. & N.A. & N.A. & N.A. & N.A. \\
\hline 2 & TRIC00027_1103 & Female (F1), diploid, WGA & 9.4 & 0.17 & 1128 & 164.6 & 774,450 & 595,204 & 43,763 & 42,062 \\
\hline 2 & TRIC00027_1199 & Pool of haploid males (F2) $(\mathrm{n}=229)$, no WGA & 359.6 & N.A. & N.A. & 270.6 & $5,878,301$ & $4,437,984$ & 127,380 & 125,302 \\
\hline 3 & TRIC00027_3101 & Male (F0), haploid, WGA & Too low & N.A. & N.A. & N.A. & N.A. & N.A. & N.A. & N.A. \\
\hline 3 & TRIC00027 3102 & Female (F0), diploid, WGA & 13.9 & 2.9 & 390 & 247.7 & $7,006,980$ & $5,365,499$ & 147,718 & 140,690 \\
\hline 3 & TRIC00027_3103 & Female (F1), diploid, WGA & 10.7 & 0.43 & 226 & 137.3 & $2,616,330$ & $1,991,305$ & 93,606 & 89,543 \\
\hline 3 & TRIC00027_3199 & $\begin{array}{l}\text { Pool of haploid males (F2) (n=1415), no } \\
\text { WGA }\end{array}$ & 670.7 & N.A. & N.A. & 228.4 & $7,510,904$ & $5,764,475$ & 131,267 & 129,249 \\
\hline
\end{tabular}

2 


\section{Table 2 (on next page)}

Pairwise comparison of loci obtained for females of the F1 generation and pools of males of the $F 2$ generation

Analysed loci have been first corrected by rxstacksfor genotype and haplotype calls and

filtered with populations. Only loci that were present in the two samples with a stack depth of 10 were kept. 


\section{$1 \quad$ Table 2}

\begin{tabular}{|c|c|c|c|c|c|}
\hline Pair of compared samples & Nb of shared loci & $\begin{array}{l}\text { Percentage of identical } \\
\text { loci (homozygous) }\end{array}$ & $\begin{array}{l}\text { Percentage of identical } \\
\text { loci (heterozygous) }\end{array}$ & $\begin{array}{l}\text { Percentage of loci with } \\
\text { differences possibly } \\
\text { explained by the } \\
\text { experimental setup }\end{array}$ & $\begin{array}{l}\text { Percentage of loci with } \\
\text { differences not explained } \\
\text { by the experimental setup }\end{array}$ \\
\hline \multirow{2}{*}{$\begin{array}{l}\text { In cross \#1 } \\
\text { F1Female versus pool of F2 males }\end{array}$} & \multirow[t]{2}{*}{48,189} & 97.7 & 1.3 & 0.6 & 0.4 \\
\hline & & \multicolumn{2}{|c|}{$\begin{array}{l}\text { Total percentage of identical loci } \\
99.0\end{array}$} & \multicolumn{2}{|c|}{$\begin{array}{c}\text { Total percentage of loci with differences } \\
1.0\end{array}$} \\
\hline \multirow{2}{*}{$\begin{array}{l}\text { In cross \#2 } \\
\text { F1 Female versus pool of F2 males }\end{array}$} & \multirow[t]{2}{*}{5,184} & 96.8 & 0.6 & 1.5 & 1.1 \\
\hline & & \multicolumn{2}{|c|}{$\begin{array}{l}\text { Total percentage of identical loci } \\
97.4\end{array}$} & \multicolumn{2}{|c|}{$\begin{array}{c}\text { Total percentage of loci with differences } \\
2.6\end{array}$} \\
\hline \multirow{2}{*}{$\begin{array}{l}\text { In cross \#3 } \\
\text { F1 Female F1 versus pool of F2 } \\
\text { males }\end{array}$} & \multirow[t]{2}{*}{20,095} & 98.2 & 0.3 & 0.9 & 0.6 \\
\hline & & $\begin{array}{r}\text { Total percentag } \\
9 \\
\end{array}$ & $\begin{array}{l}\text { of identical loci } \\
.5\end{array}$ & \multicolumn{2}{|c|}{$\begin{array}{c}\text { Total percentage of loci with differences } \\
1.5\end{array}$} \\
\hline
\end{tabular}




\section{Table 3(on next page)}

Summary of differences observed between F1 females and the whole progeny of the F1 generation (pool of F2 males).

For clarity, alleles are represented with capital letters A, B and C 


\begin{tabular}{|c|c|c|}
\hline & F1 female & Pool of F2 males \\
\hline \multirow{2}{*}{$\begin{array}{l}\text { Differences explained by experimental setup } \\
(60 \%)\end{array}$} & 1 allele $(\mathrm{A})$ & 2 alleles $(\mathrm{A}, \mathrm{B})$ \\
\hline & 2 alleles $(\mathrm{A}, \mathrm{B})$ & 3 alleles $(\mathrm{A}, \mathrm{B}, \mathrm{C})$ \\
\hline \multirow{5}{*}{$\begin{array}{l}\text { Differences not explained by experimental } \\
\text { setup }(40 \%)\end{array}$} & 1 allele $(\mathrm{A})$ & 1 allele (B) \\
\hline & 2 alleles $(\mathrm{A}, \mathrm{B})$ & 1 allele $(\mathrm{A})$ \\
\hline & 2 alleles $(\mathrm{A}, \mathrm{B})$ & 1 allele $(\mathrm{C})$ \\
\hline & 1 allele $(\mathrm{C})$ & 2 alleles $(\mathrm{A}, \mathrm{B})$ \\
\hline & 2 alleles $(\mathrm{A}, \mathrm{C})$ & 2 alleles $(\mathrm{A}, \mathrm{B})$ \\
\hline
\end{tabular}

1 


\section{Table 4 (on next page)}

Comparison of loci obtained for the first crossing experiment

Analysed loci have been first corrected by rxstacks for genotype and haplotype calls and filtered with populations. Only loci that were present in the four samples with a stack depth of 10 were kept. 
$1 \quad$ Table 4

\begin{tabular}{|c|c|c|c|c|}
\hline Compared samples & Nb of shared loci & $\begin{array}{l}\text { Percentage of identical } \\
\text { loci (homozygous) }\end{array}$ & $\begin{array}{l}\text { Percentage of loci consistent with } \\
\text { the experimental setup and } \\
\text { Mendelian inheritance of alleles }\end{array}$ & $\begin{array}{l}\text { Percentage of loci not consistent with } \\
\text { the experimental setup and Mendelian } \\
\text { inheritance of alleles }\end{array}$ \\
\hline $\begin{array}{l}\text { In Cross \#1 } \\
\text { - F0 female } \\
\text { - F0 male } \\
\text { - one F1 female } \\
\text { - pool of F2 males (i.e. progeny of } \\
\text { the F1 generation) }\end{array}$ & 32,913 & 97.3 & 98.8 & 1.2 \\
\hline
\end{tabular}




\section{Table 5 (on next page)}

Categories of SNPs not compatible with the mode of reproduction of the studied model (haplodiploidy) or Mendel's laws of inheritance and number of occurrences of each case.

The different situations are illustrated by examples taken from the analysis of the 385 questionable SNPs. 


\section{Table 5}

\begin{tabular}{|c|c|c|c|c|c|}
\hline Description & F0 Male & F0 Female & F1 Female & Pool of F2 males & Occurrences \\
\hline \multirow{3}{*}{ F0 Male incompatible } & A/G & G & G & G & \multirow{3}{*}{ ( $21.04 \%$ of the problematic SNPs $; 0.002 \%$ of the analysed $n t)$} \\
\hline & & & & & \\
\hline & TG & GA & GA & GA & \\
\hline \multirow{3}{*}{ F0 Female incompatible } & $\mathrm{C}$ & $\mathrm{C} / \mathrm{T}$ & $\mathrm{C}$ & $\mathrm{C}$ & \multirow{3}{*}{ ( $32.47 \%$ of the problematic SNPs $; 0.003 \%$ of the analysed $n t)$} \\
\hline & $\mathrm{A}$ & $\mathbf{A}$ & $\mathrm{A} / \mathrm{G}$ & $\mathrm{A} / \mathrm{G}$ & \\
\hline & G & $\mathbf{A}$ & G & $\mathrm{G}$ & \\
\hline \multirow{4}{*}{ F1 Female incompatible } & $\mathrm{C}$ & C & $\mathrm{A} / \mathrm{C}$ & $\mathrm{C}$ & \multirow{4}{*}{ ( $36.62 \%$ of the problematic SNPs $; 0.004 \%$ of the analysed $n t)$} \\
\hline & $\mathrm{T}$ & $\mathrm{C} / \mathrm{T}$ & $\mathbf{C}$ & $\mathrm{C} / \mathrm{T}$ & \\
\hline & GG & AG/GG & GG/GT & AG/GG & \\
\hline & $\mathrm{T}$ & $\mathrm{A}$ & $\mathbf{A}$ & $\mathrm{A} / \mathrm{T}$ & \\
\hline \multirow{4}{*}{ Pool of F2 males incompatible } & $\mathrm{T}$ & $T$ & $\mathrm{~T}$ & $C / T$ & \multirow{4}{*}{ ( $7.53 \%$; of the problematic SNPs ; $0.0008 \%$ of the analysed nt) } \\
\hline & A & $A / G$ & $A / G$ & A & \\
\hline & AA & AA & AA & $\mathbf{C C}$ & \\
\hline & $\mathrm{C}$ & $\mathrm{T}$ & $\mathrm{C} / \mathrm{T}$ & $\mathbf{C}$ & \\
\hline Combination of the different situations & $\mathbf{C} / \mathbf{G}$ & $\mathrm{C} / \mathrm{G}$ & $\mathrm{C} / \mathrm{G}$ & $\mathbf{C}$ & $\begin{array}{c}9 \\
(2.34 \% ; \text { of the problematic SNPs } ; 0.0002 \% \text { of the analysed } n t)\end{array}$ \\
\hline
\end{tabular}

2 